\title{
Coronavirus disease 2019 (COVID-19) outbreak among vaccinated staff and residents at a skilled nursing facility in Los Angeles County
}

\author{
Prabhu P. Gounder MD, MPH ${ }^{1}$ (1), Mona R. Saint MD, MPH ${ }^{1}$, Cesar A. Larios MHA, BSN, RN², Nicole J. Fountas MPH ${ }^{1}$, \\ Patrick Tran BSN, RN-BC ${ }^{1}$, Aya P. Obara MPH${ }^{1}$, Kim N. Bui MPH ${ }^{1}$, Maverick R. Sison MPH ${ }^{1}$, Faith M. Washburn MPH , \\ Elizabeth A. Cabigao LVN², Cynthia Chavez LVN² and Pingting Nie MD ${ }^{1}$ \\ ${ }^{1}$ Los Angeles County Department of Public Health, Los Angeles, California and ${ }^{2}$ Avalon Villa Healthcare, Los Angeles, California
}

To the Editor-It has been well-established that COVID-19 vaccines are highly effective at preventing symptomatic infection, hospitalization, and death. ${ }^{1-3}$ It is unknown, however, the extent to which vaccinated persons with COVID-19 can transmit severe acute respiratory coronavirus virus 2 (SARS-CoV-2) to others. We describe a COVID-19 outbreak in a skilled nursing facility (SNF) with high vaccine coverage among staff and residents.

The Los Angeles County (LAC) Department of Public Health (DPH) required weekly SARS-CoV-2 surveillance testing of all staff in SNFs. All COVID-19 cases identified in SNFs are immediately reportable to the DPH. An outbreak in an SNF was defined as $\geq 1$ case occurring among residents. A SNF with a single case in a staff or resident is required to conduct weekly response testing of all staff and residents; an outbreak is considered to have ended when no cases are identified for $\geq 14$ days. All outbreaks are investigated by the DPH to assess risk factors for transmission and to provide recommendations for control measures.

In May 2021, COVID-19 was identified in an asymptomatic, vaccinated staff member on day 0 (Fig. 1). On a recurring weekly survey conducted May 10, 2021, this SNF reported 87 residents (85\% fully vaccinated) and 99 directly employed and contract staff (67\% fully vaccinated). Response testing conducted on day 1

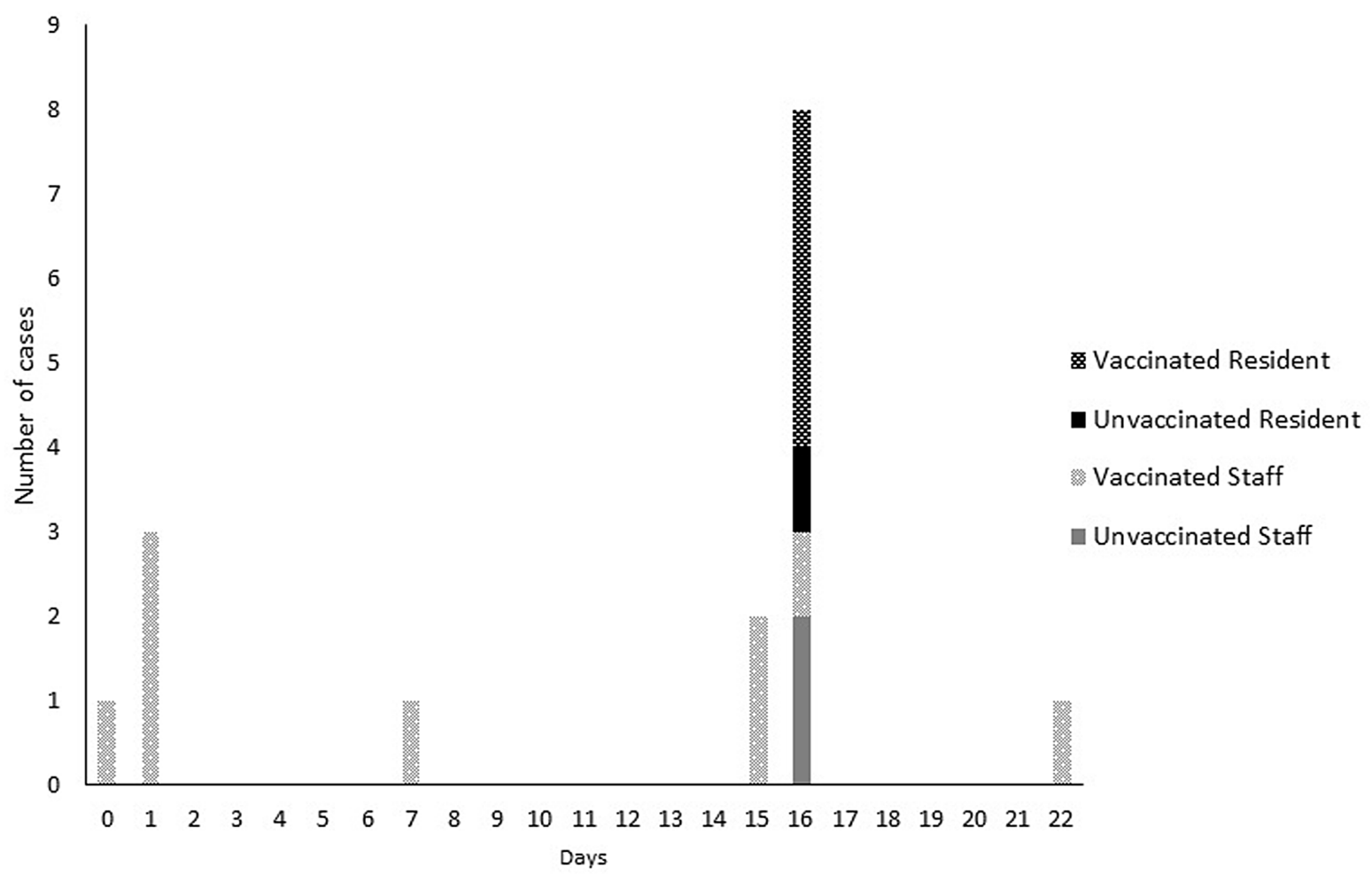

Fig. 1. Epidemic curve of COVID-19 cases in a skilled nursing facility—Los Angeles County, May-June 2021.

identified 3 cases in asymptomatic, vaccinated staff. Subsequent rounds of response testing identified additional COVID-19 infections in 7 staff members and 5 residents. Of the 7 additional staff members with COVID-19, 5 were fully vaccinated and asymptomatic, 1 was unvaccinated and asymptomatic, and 1 was unvaccinated and symptomatic (constitutional, respiratory, and gastrointestinal symptoms,
Author for correspondence: Prabhu P.Gounder, E-mail: PGounder@ph.lacounty.gov Cite this article: Gounder PP, et al. (2022). Coronavirus disease 2019 (COVID-19) Angeles County. Infection Control \& Hospital Epidemiology, 43: 1982-1983, https:// doi.org/10.1017/ice.2021.420 
and loss of taste and smell). Of the 5 residents with COVID-19, 4 were fully vaccinated and asymptomatic, and 1 was unvaccinated and asymptomatic. All 4 of the fully vaccinated residents and 8 of the 9 fully vaccinated staff members had a presumed re-infection, which we define as having a positive COVID-19 test $>90$ days prior to their current infection. In the 48 hours prior to symptom onset, the unvaccinated staff member with infection provided direct clinical care to 3 residents who developed infection and worked in proximity to the other 2 infected residents. The staff member was restricted from work upon experiencing symptoms. The nature of the residents' exposure to the other staff members with COVID-19 is unknown because their lack of symptoms precluded our ability to determine a precise infectious period. None of the infected residents left the facility or had visitors in the 21 days prior to their positive SARS-CoV-2 test. An infection control assessment by the DPH did not identify any deficiencies. The SARS-CoV-2 polymerase chain reaction (PCR) cycle threshold $(\mathrm{Ct}$ ) values ranged from 31.5 to 34 (the cutoff for a positive test at the laboratory used by the SNF), indicating low levels of virus that were insufficient for conducting whole-genome sequencing.

Our study had several limitations. First, the lack of sequencing data from outbreak-associated SARS-CoV-2 isolates meant that we could not exclude the possibility of cases resulting from multiple introductions into the facility, as opposed to transmission arising from a single common source. Second, the lack of symptoms for most outbreak-associated cases made it difficult to determine the exact onset of infection, which is necessary for determining precise incubation periods and for interpreting SARS-CoV-2 PCR Ct values. Despite these limitations, our investigation provides strong circumstantial evidence that SARS-CoV-2 was introduced into the SNF by an asymptomatic vaccinated SNF staff member who likely acquired infection in the community. The possibility that asymptomatic vaccinated persons can transmit infection carries important policy implications. Precautionary measures, such as a need for continued use of face masks by healthcare workers (regardless of vaccination status), must be implemented when there is substantial community transmission of SARS-CoV-2. Our investigation also supports continuing weekly surveillance testing, which allowed for the detection of infection in asymptomatic persons; early detection of an outbreak allows the DPH to assess and address potential gaps in infection control. All vaccinated persons were asymptomatic, and all except for one vaccinated case had evidence of prior COVID-19, indicating that immunity by past infection or vaccination can protect against symptomatic illness and severe disease. The occurrence of asymptomatic infections among fully immunized persons, however, could support the need for COVID-19 booster doses to reduce transmission among SNF staff members and residents.

Acknowledgments. We would like to thank the DPH Acute Communicable Disease Control COVID-19 Outbreak Data Team, who conducted an in-depth review of all residents at this skilled nursing facility to ensure that no other cases were identified during the facility outbreak period. We would also like to thank the COVID-19 Outbreak Management Branch members for their efforts in investigating this outbreak and collecting information on all cases.

Financial support. Data collection for this report was supported by supplementary COVID-19 funding from the US Centers for Disease Control and Prevention (grant no. 6 NU50CK000498-02-07).

Conflicts of interest. All authors report no conflicts of interest relevant to this article.

\section{References}

1. Sadoff J, Gray G, Vandebosch A, et al. Safety and efficacy of single-dose Ad26.COV2.S vaccine against COVID-19. N Engl J Med 2021;384:21872201.

2. Polack FP, Thomas SJ, Kitchin N, et al. Safety and efficacy of the BNT162b2 mRNA COVID-19 vaccine. N Engl J Med 2020;383:2603-2615.

3. Baden LR, El Sahly HM, Essink B, et al. Efficacy and safety of the mRNA-1273 SARS-CoV-2 vaccine. N Engl J Med 2021;384:403-416.

\title{
Transmission of severe acute respiratory coronavirus virus 2 (SARS-CoV-2), delta variant, between two fully vaccinated healthcare personnel
}

\author{
L. Leigh Smith $\mathrm{MD}^{1}$ (1), Aaron M. Milstone MD, MHS ${ }^{2}$ (1), Morgan Jibowu MPH ${ }^{3}$, Chun Huai Luo MS ${ }^{4}$, \\ C. Paul Morris MD, PhD ${ }^{4,5}$, Heba H. Mostafa MD, PhD ${ }^{4}$ and Lisa L. Maragakis MD, MPH ${ }^{1}$
}

${ }^{1}$ Division of Infectious Diseases, Department of Medicine, Johns Hopkins University School of Medicine, Baltimore, Maryland, ${ }^{2}$ Division of Infectious Diseases, Department of Pediatrics, Johns Hopkins University School of Medicine, Baltimore, Maryland, ${ }^{3}$ Department of Healthcare Epidemiology and Infection Control, Johns Hopkins Health System, Baltimore, Maryland, ${ }^{4}$ Division of Medical Microbiology, Department of Pathology, Johns Hopkins Hospital, Baltimore, Maryland and ${ }^{5}$ National Institute of Allergy and Infectious Disease, National Institutes of Health, Bethesda, Maryland

To the Editor-Breakthrough severe acute respiratory coronavirus virus 2 (SARS-CoV-2) infection causing coronavirus disease 2019 (COVID-19) in fully vaccinated individuals occurs, and the frequency is increasing since the SARS-CoV-2 delta variant virus

\footnotetext{
Author for correspondence: L. Leigh Smith, E-mail: 1smit213@jh.edu
}

Cite this article: Smith LL, et al. (2022). Transmission of severe acute respiratory coronavirus virus 2 (SARS-CoV-2), delta variant, between two fully vaccinated healthcare personnel. Infection Control \& Hospital Epidemiology, 43: 1983-1985, https://doi.org/10.1017/ice.2021.469 began circulating widely. ${ }^{1}$ COVID-19 vaccines are highly effective at reducing SARS-CoV-2 shedding and transmission. ${ }^{2}$ The question of whether fully vaccinated people with breakthrough COVID-19 can transmit the SARS-CoV-2 virus to others is central to the debate around the need for mitigation efforts including masking and physical distancing for fully vaccinated individuals. We report apparent SARS-CoV-2 viral transmission between 2 fully vaccinated healthcare workers $(\mathrm{HCW})$ in the setting of occupational unmasked close contact. 